\title{
Protective Effect of Ad-VEGF-Bone Mesenchymal Stem Cells on Cerebral Infarction
}

\author{
Bo $\mathrm{CHEN}^{1 *}$, Feng ZHANG ${ }^{2}$, Qiao-Yu LI ${ }^{3}$, Aihua GONG ${ }^{4}$, Qing LAN ${ }^{1}$ \\ ${ }^{1}$ The Second Hospital Affiliated to Soochow University, Department of Neurosurgery, Soochow, China \\ ${ }^{2}$ The 101 Hospital of Chinese People's Liberation Army, Department of Neurosurgery, Wuxi, China \\ ${ }^{3}$ The First People's Hospital of Zhenjiang, Department of Neurosurgery, Zhenjiang, China \\ ${ }^{4}$ Jiangsu University, School of Clinical Medicine, Zhenjiang, China \\ *Bo CHEN and Feng ZHANG contributed equally to this work and are joint first authors.
}

To watch the surgical videoclip, please visit http://turkishneurosurgery.org.tr/images/11488-video.mp4

\section{ABSTRACT}

AIM: To understand the mechanism of intracerebroventricular transplantation of vascular endothelial growth factor (VEGF) genemodified bone mesenchymal stem cells (BMSCs) in rats after cerebral infarction.

MATERIAL and METHODS: The middle cerebral artery occlusion ischemia/reperfusion (MCAO I/R) model was established in rats using the Zea-Longa suture method. A recombinant adenovirus (Ad-VEGF) was engineered to express VEGF. The rats were divided into 3 groups. Control BMSC infected with control adenovirus (BMSC-Ad), BMSC infected by Ad-VEGF (BMSC-Ad-VEGF), and phosphate buffered saline (PBS) suspension were injected into the intracerebroventricular system of the rats in groups 1, 2 and 3 respectively, 24 hours after middle cerebral artery occlusion (MCAO). The neurological function of rats was evaluated with the modified Neurological Severity Scores (mNSS). The infarct volume of brain in rats was determined using 2,3,5-triphenyltetrazolium chloride (TTC) stain at 14 days. GFAP and pGSK3 $\beta$ expression of ischemic penumbra was determined using immunohistochemical method. GFAP, pAKT, AKT, and pGSK3 $\beta$ expressions were determined with Western blot.

RESULTS: Functional improvement was accelerated in animals receiving BMSC-Ad, while improvement at all times between 7 days and 28 days post MCAO was significantly greater in animals transplanted with BMSC-Ad-VEGF than for other treated animals. The number of GFAP-labeled cells was prevented by post-ischemic BMSC-Ad-VEGF treatment; pMCAO activate the PI3K/AKT/GSK3 $\beta$ pathway to reduce reactive gliosis.

CONCLUSION: Our findings demonstrate that PI3K/AKT/GSK3 $\beta$ pathway could reduce reactive gliosis, ameliorate neurological deficit, diminish the percentage of cerebral infarction volume in rats, and facilitate angiogenesis.

KEYWORDS: Bone mesenchymal stem cell, Vascular endothelial growth factor, Ischemic stroke, Intracerebroventricular injection, Phosphoinositide-3-kinase/Akt /glycogen synthase kinase-3 $\beta$ pathway 
Chen B. et al: Protective Effect of Stem Cells on Cerebral Infarction

\section{INTRODUCTION}

Despite the development of medical technology, cerebral vascular disease (CVD), approximately $70 \%$ of which is cerebral infarction, remains as the major cause of death and disability worldwide $(20,23)$. The tissue-type plasminogen activator substance, which is used to treat cerebral infarction, is the only drug approved by the U.S. Food and Drug Administration. However, given the secondary bleeding risk induced by thrombolytic therapy, only $3 \%$ of the patients with cerebral infarction can be treated with thrombolytic agents $(11,24)$. Based on its multi-directional differentiation and proliferation ability, transplanting bone mesenchymal stem cells (BMSCs) for CVD treatment has attracted increasing attention. More importantly, these cells can secrete various cytokines and neurotrophic factors, such as nerve growth factor (NGF) and brain-derived neurotrophic factor (BDNF) that can promote ischemia brain functional recovery (4). Vascular endothelial growth factor (VEGF), which can promote endothelial cell proliferation and angiogenesis, is important in nerve cell protection $(6,7)$. Moreover, some studies have suggested that PI3K/AkT/GSK3 $\beta$ could affect neuronal death in rat cerebral ischemia models of ischemia reperfusion, but the mechanisms of how to improve the ischemic injury remains unclear $(8,14,18)$. In the present study, we aimed to investigate the therapeutic potential of the VEGF-BMSCs in cerebral ischemia of the rats.

\section{MATERIAL and METHODS}

\section{Experimental Reagents and Materials}

Ethical approval for this study was obtained from the Ethical Committe of The Second Hospital affiliated to Soochow University. Ratmesenchymalstemcellosteogenic differentiation medium (CYAGEN, CHINA), $\beta$-glycerophosphate, vitamin C, dexamethasone (Sigma), inverted phase contrast microscope (NIKON, Japan), medical clean bench (Jiangsu Sujing, China), adenovirus vector carrying human VEGF (Li Huiyong cooperative construction), stereotaxic instrument (Clinical Medical College of Jiangsu University), 2,3,5-3-triphenyl tetrazolium chloride (TTC, Amresco), 10\% chloral hydrate, fishing line, anti-rat VEGF antibody (SANTA), anti-rat GFAP antibody (SANTA), anti-rat AKT antibody (Wuhan Boster), anti-rat pAKT antibody (Santa), rat GSK (Cell Signaling), and rat pGSK (Cell attracted increasing attention Signaling) were used.

\section{METHODS}

\section{Rat BMSCs Culture}

Approximately $100 \mathrm{~g}$ femur and tibia of male Sprague-Dawley (SD) rats were removed from the epiphyseal side, and then the femoral and tibial bone marrow cavity was flushed with L-DMEM culture. Then, the suspension was directly inoculated in the culture bottle and cultured in L-containing $10 \%$ foetal bovine serum in DMEM (FBSL-DMEM) at $37^{\circ} \mathrm{C}$ in a humidified atmosphere with $5 \% \mathrm{CO}_{2}$. The medium was first changed after $24 \mathrm{~h}$, and changed again after $48 \mathrm{~h}$ to remove non-adherent blood cells. The medium was then changed every $3 \mathrm{~d}$. The
BMSCs were passaged at ratio 1:2. When passaged to the third generation cells, pure rat BMSCs were obtained.

\section{Expression of Rat BMSC Markers}

Second-generation of rat BMSCs were digested and passaged into 24-well plate species. After culturing for $3 \mathrm{~d}$, the BMSCs were treated with CY3-labeled rabbit anti-mouse CD44, CD105, CD133, and vimentin. The cells were observed under the fluorescence microscope and photographed. Nuclei were labelled with Hoechst 33342. PBS was used as a negative control instead of primary or secondary antibody.

\section{Osteogenic and Adipogenic Differentiation of Rat BMSCS}

Osteogenic culture: Third-generation rat BMSCs were seeded at $2 \times 104$ cells/well in six-well plate under osteogenic conditions: DMEM $(L)+10 \%$ foetal bovine +10 $\mathrm{mmol} / \mathrm{L} \beta$-glycerophosphate $+1 \times 10-6 \mathrm{~mol} / \mathrm{L}$ dexamethasone $+50 \mathrm{mg} / \mathrm{L}$ ascorbic acid. The medium was changed every $3 \mathrm{~d}$, induced for $14 \mathrm{~d}$, and calcium nodules were observed with the use of alizarin red $\mathrm{S}$ staining.

Adipogenic culture: The third-generation rat BMSCs were seeded at $2 \times 104$ cells/well in $2 \mathrm{~mL}$ of induced lipid A medium and incubated for $3 \mathrm{~d}$, and then changed to $2 \mathrm{~mL}$ of induced lipid B medium, which was changed after $1 \mathrm{~d}$. This process was repeated thrice. The cells were stained with oil red $\mathrm{O}$, washed thrice with PBS, and then observed under inverted microscope. The cells containing stained red fat particles were recorded as adipogenic-positive cells.

\section{Transplantation Procedure}

Approximately 24 hours after SD rat model establishment (Figure 1A, B), the rats with neurological score from levels I to III were randomly divided into 3 groups (each $n=20$ ) and received intracerebroventricular inoculation of phosphate buffered saline (PBS), BMSC-Ad or BMSC-Ad-VEGF: Group 1 received PBS, Group 2 received BMSC infected with control adenovirus (BMSC-Ad) and Group 3 received BMSC infected by Ad-VEGF (BMSC-Ad-VEGF). A total of 60 SD rats were anesthetized with chloral hydrate $(3 \mathrm{~mL} / \mathrm{kg}$ intraperitoneal) and transferred to a stereotaxic apparatus (Figure 1B). Using aseptic technique $1 \mathrm{~cm}$ incision was made in the scalp 1.5 $\mathrm{mm}$ lateral to the bregma. Using a dental drill a burr hole was made in the bone $1.5 \mathrm{~mm}$ lateral to the bregma and the following suspension were slowly injected over 20 minutes into the lateral ventricle at a depth of $4.5 \mathrm{~mm}$ from the surface of the brain: Group 1: $20 \mu \mathrm{L}$ PBS; Group 2: BMSC- Ad $\left(1 \times 10^{6}\right.$ cells); Group 3: BMSC-Ad-VEGF $\left(1 \times 10^{6}\right.$ cells $)$.

\section{Measurement of Infarct Volume}

2,3,5-triphenyltetrazolium chloride (TTC) staining was employed to measure infarct volume. Five rats from each group were taken $14 \mathrm{~d}$ after cell transplantation and then sacrificed by anaesthesia overdose. Whole brain was removed and placed in the freezer for $10 \mathrm{~min}$ at $-20^{\circ} \mathrm{C}$. Infarct volume was calculated using the formula $\mathrm{V}=t(\mathrm{~A} 1+\mathrm{An})-(\mathrm{A} 1+\mathrm{An}) / 2$ ( $t$ is the slice thickness, and $A$ is infarction area). The volume of contralateral cerebral of infarction was calculated using the same method. The results was then calculated as infarct volume divided by contralateral cerebral of infarction volume. 

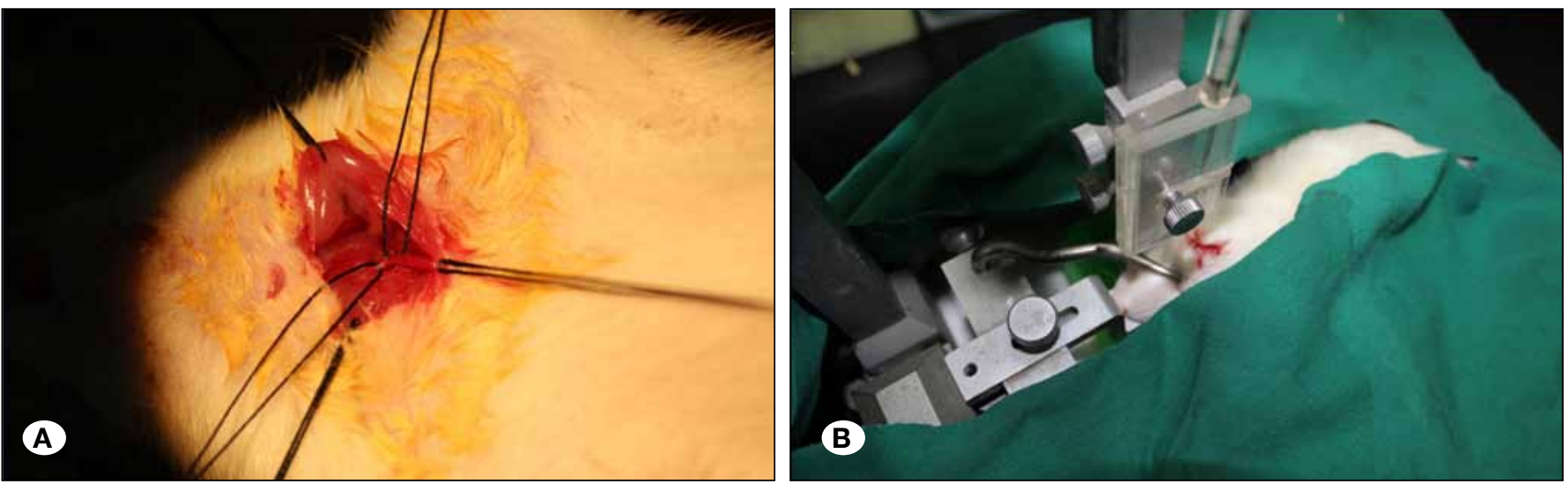

Figure 1: A) Establishment of MCAO I/R injury in rat using Zea-Longa suture method. B) Inoculation of PBS, BMSC-Ad and BMSC-AdVEGF using stereotactic method.

\section{Behavioral Testing}

Nerve function of each rat group was evaluated using modified neurological Severity Score (mNSS) (16) over 4 weeks (Figure $2 \mathrm{~A}-\mathrm{F}$ ). The mNSS is a composite of motor (muscle status, abnormal movement), sensory (visual, tactile, proprioceptive), reflex, and balance tests. Neurological function is graded on a scale of 0 to 18,0 , normal; 1 to 6 , mild injury; 7 to 12 , moderate injury; 13 to 18 , serious damage; and 18 , most severe neurological deficits.

\section{Histological Analysis}

Five rats from each group were taken 14 days after cell transplantation and anaesthetised with the use of $10 \%$ chloral hydrate. The whole brain was removed and postfixed in $4 \%$ paraformaldehyde. Streptavidin-peroxidase was used to detect the GFAP and pGSK3 $\beta$ expression. Rabbit antirat GFAP and pGSK3 $\beta$ (1:400) antibodies were incubated overnight at $4{ }^{\circ} \mathrm{C}$, and then washed thrice with PBS, added with $\mathrm{DAB}$, stained with haematoxylin, dehydrated, sealed in transparent and neutral gum, and then observed under light microscope.

\section{Western Blotting}

For in vitro detection, the transfected and untransfected cells were collected, washed with PBS, and $50 \mu \mathrm{L}$ lysis buffer added. After protein quantification using BCA method, buffer was added into the sample. The protein samples were boiled for 5 minutes to 10 minutes and then stored at $-20{ }^{\circ} \mathrm{C}$ after aliquots were obtained.

For in vivo detection, the brains were removed, followed by left penumbra separation (100 g), which was placed in the grinder. Then $1 \mathrm{~mL}$ lysis buffer was added, and the penumbra was ground and treated with equal volume of sample buffer after protein quantification using BCA. The protein samples were boiled for 5 minutes to 10 minutes and stored at $-20{ }^{\circ} \mathrm{C}$ after aliquots were obtained. After electrophoresis, the proteins were transferred to polyvinylidene fluoride membrane, and then sealed with 5\% BSA for 1 hour at room temperature. Antibody was added first, and the samples were incubated overnight, followed by washing with TBST. The horseradish peroxidase-labelled secondary antibodies were then added, incubated for 1 hour, and washed by TBST and exposed to X-ray film.

\section{Statistical Analysis}

All data were expressed as means \pm standard deviation (SD). Data were evaluated with repeated measures of analysis of variance (ANOVA), and the unpaired Student's t-test. Where data were normally distributed the Kolmogorov-Smirnov test was applied; in other cases the Mann-Whitney U-test was applied. The significance level was set at $\mathrm{P}<0.05$.

\section{RESULTS}

\section{The Expression of VEGF in BMSC and Its Marker Expression and Induction of Osteogenesis and Adipocyte in Vitro}

Immunofluorescence of 3 days cultured primary BMSCs showed positive CD44, CD105, CD133, and vimentin expression (Figure 3A). After adipocytic differentiation and oil red $\mathrm{O}$ staining, red fat particles were found in cells, which may be determined as fat cells. The cells became square or irregular in shape after osteogenic differentiation and then gradually transformed into osteoblasts, followed by round mineralised nodule formation. Meanwhile, calcium nodule formation was confirmed as alizarin red staining was positive (Figure 3B $(a, b))$. The results indicated that BMSCs with different functions were obtained.

Western blotting of BMSC-Ad-VEGF revealed a specific protein band at $46 \mathrm{kDa}$ consistent with the predicted size of the VEGF protein. Little VEGF band was detected either in Ad-infected or uninfected cells. This experiment indicates that Ad-VEGF infects BMSC and efficiently directs the expression of VEGF protein (Figure 3C).

\section{mNSS}

There were no significant differences among the groups in NSS scores before MCAO or at day 1 after MCAO. Behavioral deficits showed progressive recovery in all groups from week 1 to week 4 after MCAO. At 14 days to 28 days after 

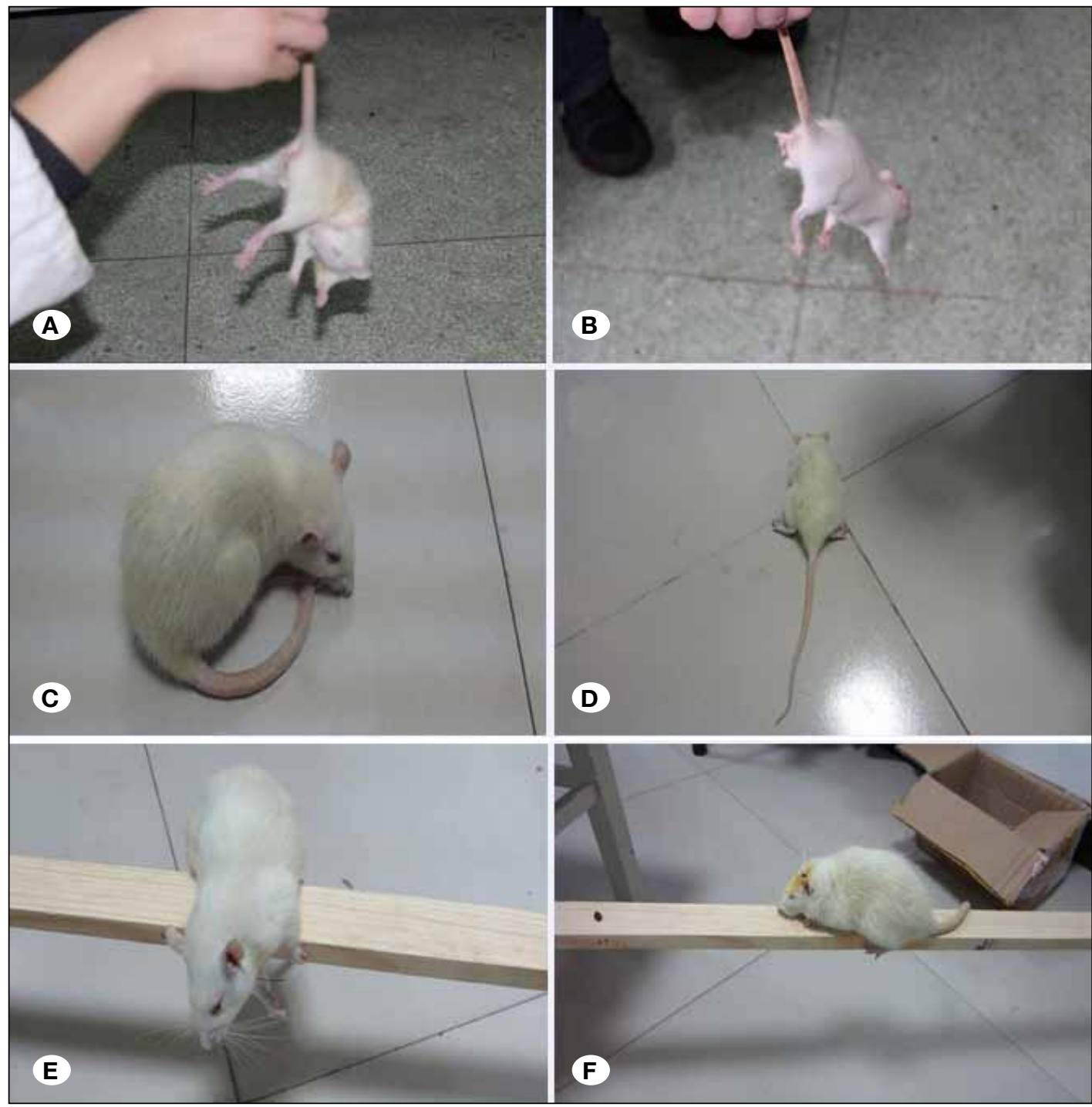

Figure 2:

A, C, E) Neurological assessment of the rats after MCAO I/R injury.

B, D, F) After the injection of BMSC-AdVEGF.
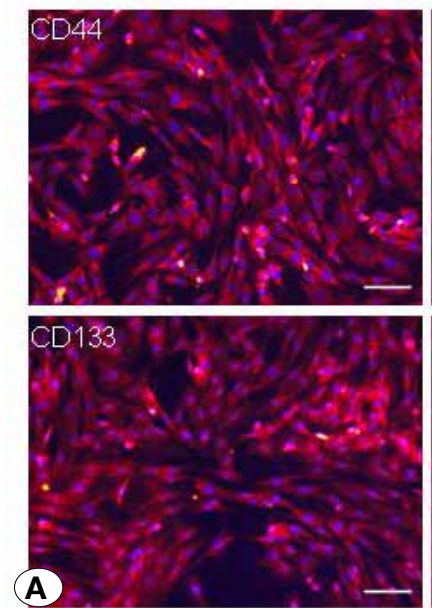
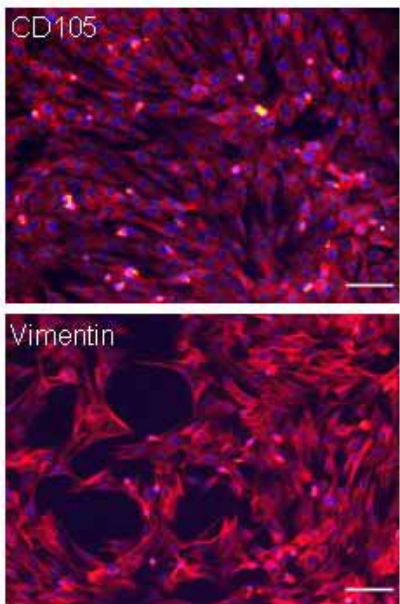

(B)
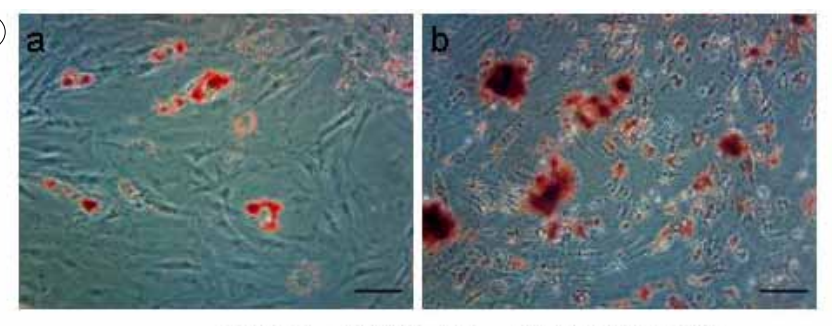

(C)

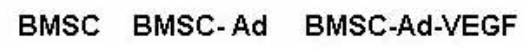

VEGF

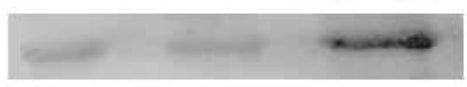

$\beta$-actin

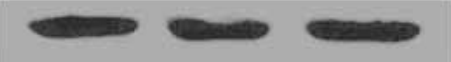

Figure 3: A) The surface markers of Bone marrow-derived mesenchymal stem cell, B) a: Adipogenic differentiation of BMSCs at 14 day.

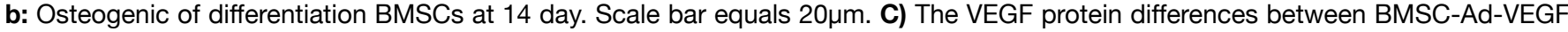
and BMSC-Ad,PBS. 
cell transplantation, compared with the PBS group, rat NSS scores of BMSC-Ad-VEGF and BMSC-Ad groups were significantly low $(\mathrm{P}<0.05)$. However, rats receiving BMSC-AdVEGF showed greater functional recovery than rats receiving BMSC-Ad ( $P<0.05$, Figures $2 A-F, 4)$ (Video 1 and 2).

\section{Measurement of infarct and HE Staining}

At 7 days after cell transplantation, the area percentage of infarct in BMSC-Ad-VEGF group $(28.12 \pm 1.73)$ was significantly lower than those of the PBS $(40.46 \pm 1.80)$ and BMSC-Ad group $(35.30 \pm 1.60)(P<0.05$, Figures $5 A, B)$.

At 7,14 , and 21 days after cell transplantation, brain tissue sections were prepared in each group followed by H\&E staining. Brain tissues in each group showed oedema and disorderly morphological structure after 7 days. Moreover, a large number of inflammatory cell infiltrations were found. Inflammatory cells were found after 14 days, which had greater remission, and tissue morphology was restored to normal. No significant difference in tissue morphology was found after 14 and 21 days (Figure 5C).

\section{BMSC-Ad-VEGF Activate pAkt/Akt and pGSK3 $\beta$ and Inhibit reactive Gliosis}

Our findings are consistent with previous reports (9), in which the Western blot results showed that the activity of pAkt was upregulated after MCAO in BMSC-Ad-VEGF group compared with those in the other groups (Figure 6B).

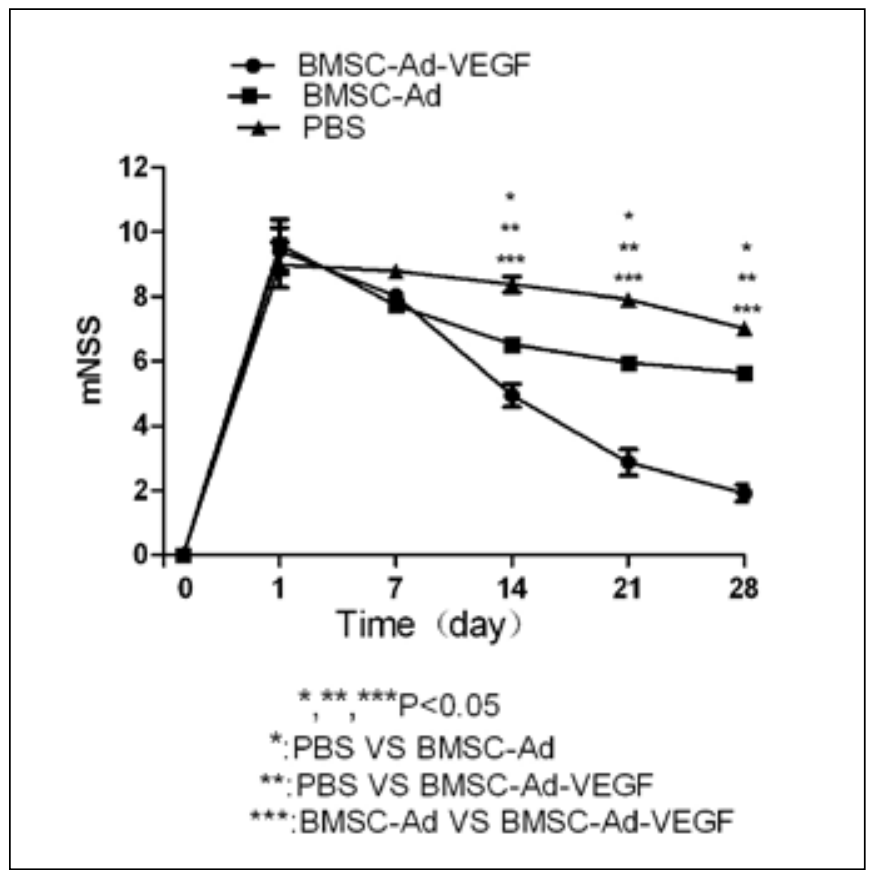

Figure 4: Nerve functions were evaluated through modified Neurological Severity Scores (mNSS). Compared with the other group, the rats' mNSS scores of BMSC-Ad-VEGF group were lower, the recovery of nerve function was the best $(P<0.05)$.

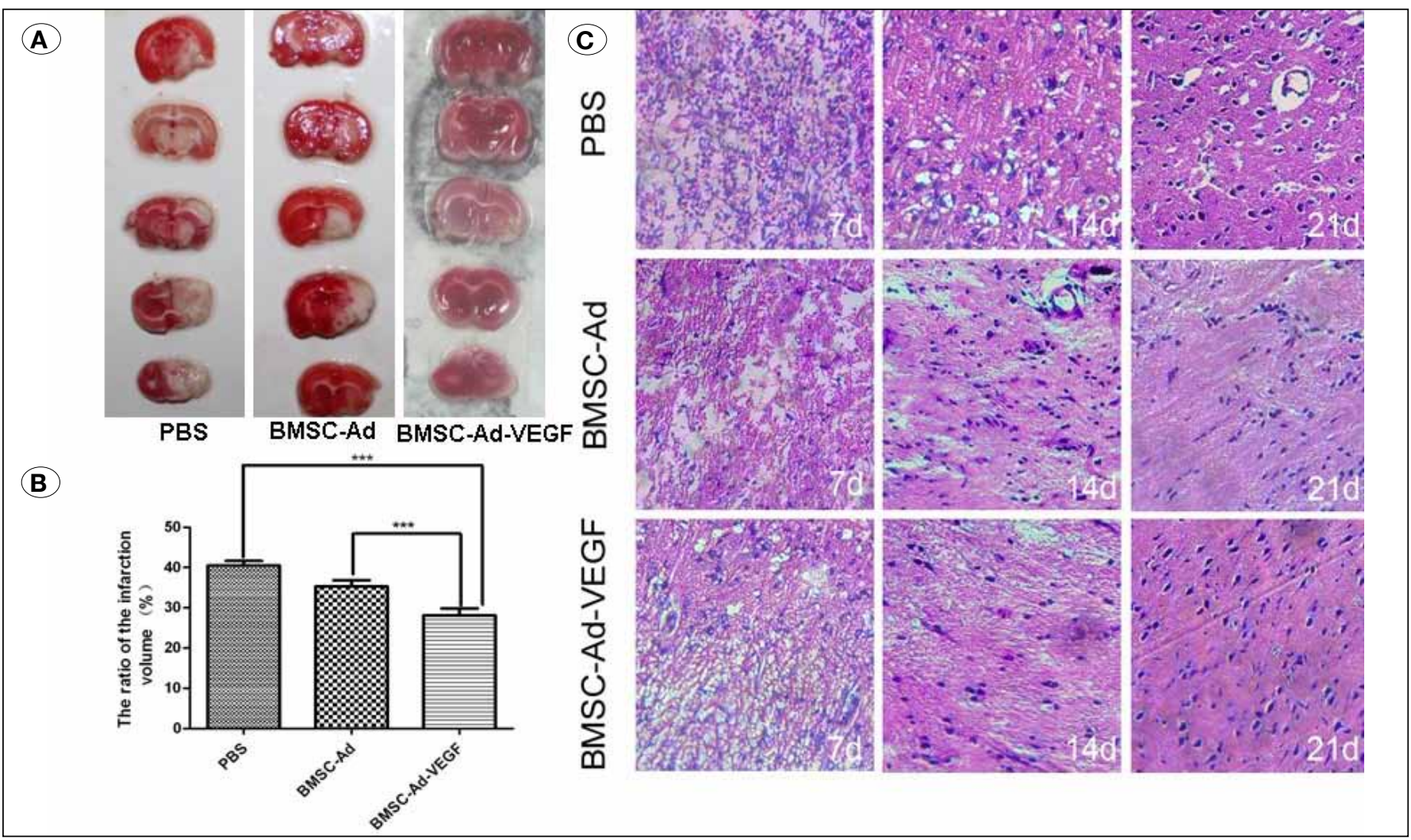

Figure 5: A) The infarct volume was measured by TTC; B) The histogram showed: Compared with the other group, The infarct volume about BMSC-Ad-VEGF group were lower $(\mathrm{P}<0.0001)$. C) The PBS, BMSC-Ad group, BMSC-Ad-VEGF group at $7 d$, 14d and 21d respectively. 
After MCAO, glial cells were activated, followed by reactive gliosis, which is mainly dependent on the degree of damage (1). To evaluate the effects after treatment of MCAO by intracerebroventricular injection of BMSC-Ad-VEGF, GFAP was used to label and count the microglia and astrocytes. GFAP-positive cells are astrocytes, stellate-shaped, protrusion slender, and deeply stained. These cells gradually increased from the central ischemic area to the ischemic penumbra, and counted under 200x magnification microscope. The results are as follows: BMSC-Ad-VEGF group (36.89 \pm 2.60$)$, BMSCAd group (48.51 \pm 1.18$)$, PBS group $(63.84 \pm 4.04)$. Statistically significant difference was found between the BMSC-Ad and PBS groups when compare with the BMSC-Ad-VEGF group $(p<0.0001$, Figure $6 A)$.

pGSK3 $\beta$ and GFAP protein expression after 1,7 , and 14 days were analysed using Western blot. No significant difference was found between each group after 1 day. However, pGSK3 $\beta$ protein expression was upregulated and GFAP protein expression was downregulated after 7 and 14 days in BMSCAd-VEGF group compared with those in the other groups (Figure 6B).

\section{DISCUSSION}

In recent years, more and more researches have interesting in the transgenic stem cell treatment of cerebral ischemia. Neural and embryonic neural stem cells, as well as BMSCs, are candidates for transgenic stem cells. BMSCs are particularly preferred as transgenic carriers for treatment of cerebral ischemia because of its accessibility, low immunogenicity and no ethical conflict.

In the current experiment, intracerebroventricular injection of BMSC-Ad-VEGF was used to treat cerebral infarction of rats. Large amounts of inflammatory mediators and chemokines

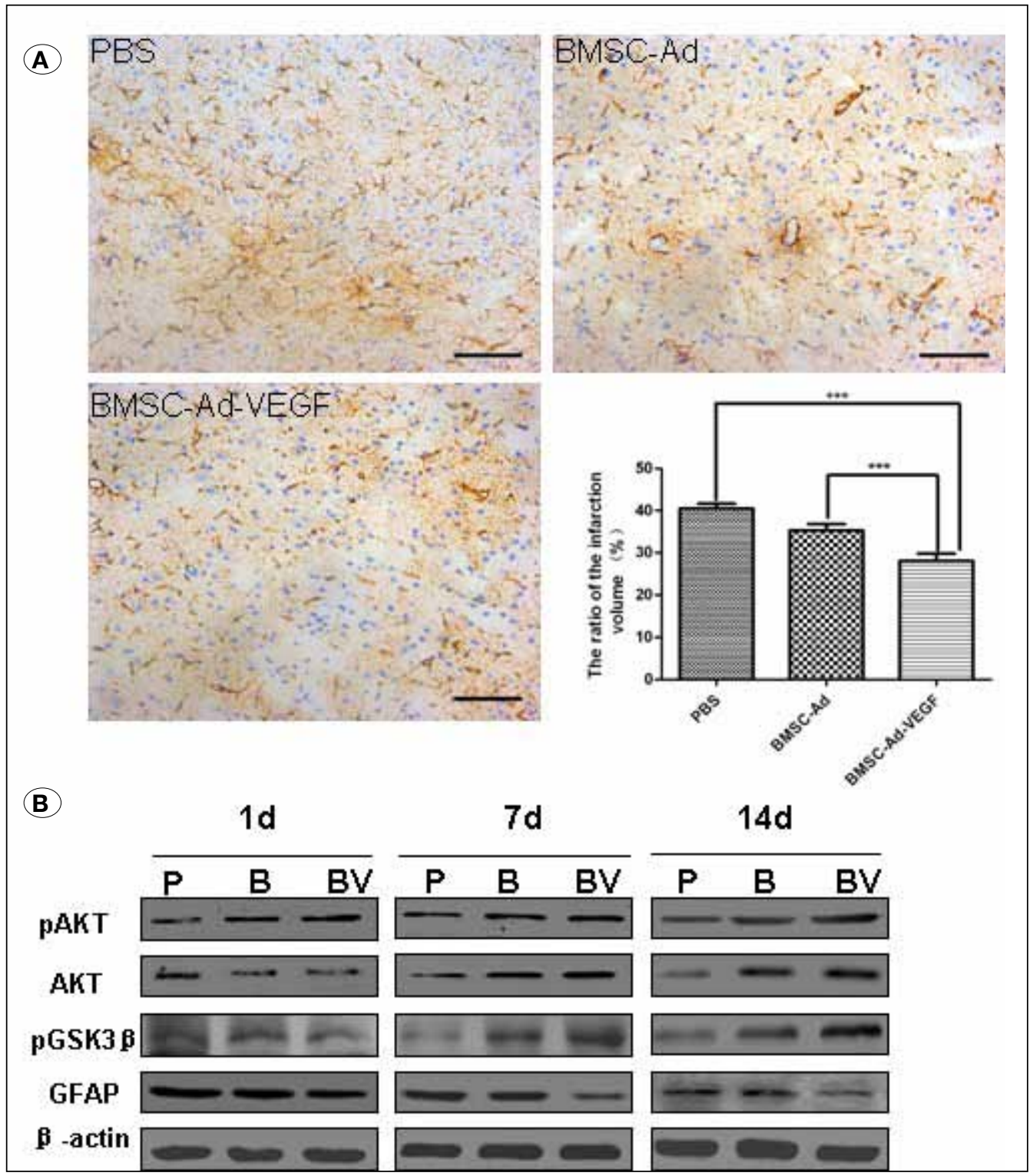

Figure 6: A) The immunohistochemical analysis showed that the reactive gliosis were attenuated after the treatment of BMSCAd-VEGF group (x200). The histogram showed the number of GFAP-positive cells. Compared with the other group, the number of GFAPpositive cells were lower after the treatment of BMSCAd-VEGF group $(p<0.0001)$ Scale bar equals $100 \mu \mathrm{m}$. B) The expression of pAKT, AKT, pGSK3 $\beta$ and GFAP were detected by western blotting. The results showed that the proportion of pAkt/ Akt was increased on BMSCAd-VEGF group. There was no significant difference in the expression of pGSK3 $\beta$ and GFAP in 1 day. Otherwise, we founded that the expression of pGSK3 $\beta$ was increased, but the expression of GFAP were lower after the treatment of BMSC-Ad-VEGF group at 7, 14 days (P: PBS; B: BMSCAd; BV: BMSC-Ad-VEGF). 
were released because of ischemic brain tissue, which facilitate the migration of BMSCs into the lesion, delivery of VEGF, and ultimately the stimulation of VEGFR2 expression $(13,20)$. This process contributed to the formation and growth of new capillaries in ischemic tissue, which reduced neuronal apoptosis and infarct size as well as promoted recovery of neurological function. Meanwhile, some nutritional factors and cytokines (such as brain-derived neurotrophic factor, nerve growth factor) were secreted in the surrounding brain tissue through paracrine and autocrine (17), which promoted stem cells to differentiate into neural-like and glial-like cells (15), instead of damaged nerve cells, thereby increasing recovery of neurological function.

Zhang et al. (23) showed that VEGF facilitates the blood brain damages to become heavier in the early stage of cerebral infarction (1 hour infarction), increases vascular permeability, and aggravates cerebral oedema and neurological damage. However, after 48 hours of cerebral infarction, the ischemic penumbra neovascularisation and recovery of nerve regeneration could be promoted by VEGF. Therefore, intracerebroventricular injection of BMSC-Ad-VEGF was chosen for the treatment of cerebral infarction in rats after 48 hours. However, HE staining showed that oedema still existed in the BMSCAd-VEGF, BMSC-Ad, and PBS groups after 7 days. These phenomena may have been caused by further exacerbation of the brain damage followed by increasing oedema due to intracerebroventricular injection. Alleviated encephaloedema, however, the PBS group still showed oedema and disordered structure. As a result, the indexes were detected after 14 days.

The signalling pathway mechanism of BMSC-Ad-VEGF treatment of cerebral infarction was studied. We found that nerve injury after ischemia could be alleviated through activation of AKT signalling pathway. Our studies indicated that the reactive gliosis increased because of the ischemic injury. Intracerebroventricular injection of BMSC-Ad-VEGF could inhibit the GFAP expression, reduce the reactive glial proliferation, activate pAKT/AKT signalling pathway, and activate the expression of downstream genes pGSK3ß. In addition, VEGF/VEGFR2/FIk1 and pAKT/AKT signalling pathway was affected by some nutritional factors secreted in the BMSC group $(5,11)$, which participated in neuroprotection. The mNSS showed significant recovery of nerve functions in the BMSC-Ad group compared with the PBS group. Significant difference was found between the BMSC-Ad and BMSC-Ad-VEGF groups. This result indicated that VEGFBMSC combination therapy stimulated the VEGF/VEGFR2/ Flk1 and pAKT/AKT signalling pathway and reduced reactive nerve gliosis, which is related to microgliastrocytes $(2,3$, 12) and ischemic damage and recovery. Therefore, it can be considered as an evaluation indicator of cerebral ischemia. The number of microglia is believed to be related to the area of cerebral infarction (24).

pAKT, an important part of the PI3K signalling pathway, thereby enhancing cell survival after activation through GSK3 and other downstream gene regulation. In various cerebral ischemia animal models, neuroprotective effects can be achieved after Akt activation. pAkt is mainly expressed in the peripheral region of the core damage, but less in severely damaged core area, which is related to inadequate energy to activate Akt. Glycogen synthase kinase 3 (GSK3), which is involved in glucose metabolism, inhibits glycogen synthesis. GSK3 is mainly divided into two subtypes, namely, GSK3a and GSK3 $\beta$. GSK3 $\beta$ is mainly present in the nervous system.

Endo et al. (8) showed that in the transient cerebral ischemia rat model, GSK3 $\beta$ is expressed in the ischemic region. However, GSK3 $\beta$ expression is different in some reported animal model of cerebral ischemia, which may be associated with the degree of damage of the animal model. GSK3 $\beta$ can be activated by serious damage, which leads to apoptosis, but it can be inactivated by mild injury to promote cell survival. In the current study, the effects of PI3K/AkT/GSK3 $\beta$ signal pathway on BMSC-Ad-VEGF combined treatment of cerebral ischemia had been examined. Compared with the other groups, the p-AkT and p-GSK3 $\beta$ in the BMSC-AdVEGF group were significantly increased after treatment, and GFAP expression was reduced, followed by reduced gliosis, which resulted in nerve protection and promotion of recovery of neurological function. However, this process is only one protection mechanism against ischemic injury. Further studies on other protection mechanisms are necessary.

\section{- REFERENCES}

1. Araujo M, Wandosell F: Differential cellular response after glutamate analog hippocampus damage. J Neurosci Res 44: 397 - 409, 1996

2. Benakis $\mathrm{C}$, Bonny $\mathrm{C}$, Hirt L: JNK inhibition and inflammation after cerebral ischemia. Brain Behav Immun 24:800-811, 2010

3. Bovolenta P, Wandosell F, Nieto-Sampedro M: CNS glial scar tissue: A source of molecules which inhibit central neurite outgrowth. Prog Brain Res 94:367 - 379, 1992

4. Chen J, Li Y, Wang L, Lu M, Zhang X, Chopp M: Therapeutic benefit of intracerebral transplantation of bone marrow stromal cells after cerebral ischemia in rats. J Neurol Sci 189:49-57, 2001

5. Chiba Y, Sasayama T, Miyake S, Koyama J, Kondoh T, Hosoda $\mathrm{K}$, Kohmura E: Anti-VEGF receptor antagonist (VGA1155) reduces infarction in rat permanent focal brain ischemia. Kobe $\mathrm{J}$ Med Sci 54:E136-146, 2008

6. Connolly DT, Heuvelman DM, Nelson R, Olander JV, Eppley BL, Delfino JJ, Siegel NR, Leimgruber RM, Feder J: Tumor vascular permeability factor stimulates endothelial cell growth and angiogenesis. J Clin Invest 84:1470-1478, 1989

7. Connolly DT, Olander JV, Heuvelman D, Nelson R, Monsell R, Siegel N, Haymore BL, Leimgruber R, Feder J: Human vascular permeability factor. Isolation from U937 cells. J Biol Chem 264:20017-20024, 1989

8. Endo $\mathrm{H}$, Nito $\mathrm{C}$, Kamada $\mathrm{H}$, Nishi T, Chan PH: Activation of the Akt/GSK3beta signaling pathway mediates survival of vulnerable hippocampal neurons after transient global cerebral ischemia in rats. J Cereb Blood Flow Metab 26:1479 - 1489, 2006

9. Gao X, Zhang H, Takahashi T, Hsieh J, Liao J, Steinberg GK, Zhao $\mathrm{H}$ : The Akt signaling pathway contributes to postconditioning's protection against stroke; The protection is associated with the MAPK and PKC pathways. J Neurochem 105:943-955, 2008

10. Graham GD: Tissue plasminogen activator for acute ischemic stroke in clinical practice: A meta-analysis of safety data. Stroke 34: 2847-2850, 2003 
11. Kilic E, Kilic U, Wang Y, Bassetti CL, Marti HH, Hermann DM: The phosphatidylinositol-3 kinase/Akt pathway mediates VEGF's neuroprotective activity and induces blood brain barrier permeability after focal cerebral ischemia. FASEB $\mathrm{J}$ 20:11851187,2006

12. Kriz J: Inflammation in ischemic brain injury: Timing is important. Crit Rev Neurobiol 18(1-2):145-157, 2006

13. Laurer HL, Mcintosh TK: Experimental models of brain trauma. Curr Opin Neurol 12(6):715-721, 1999

14. Noshita N, Lewen A, Sugawara T, Chan PH: Evidence of phosphorylation of Akt and neuronal survival after transient focal cerebral ischemia in mice. J Cereb Blood Flow Metab 21:14421450,2001

15. Sarkar D, Zhao W, Gupta A, Loh WL, Karnik R, Karp JM: Cell surface engineering of mesenchymal stem cell. Methods Mol Biol 698:505-523,2011

16. Schallert T, Kozlowski DA, Humm JL, Cocke RR: Use-dependent structural events in recovery of function. Adv Neurol 73:229238,1997

17. Seung-Wan Y, Sung-Soo K, Lee SY, Lee HS, Kim HS, Lee $\mathrm{YD}$, Suh-Kim H: Mesenchymal stem cells promote proliferation of endogenous neural stem cells and survival of newborn cells in a rat stroke model. Exp Mol Med 40(4):387-397, 2008
18. Sun B, Chen L, Wei X, Xiang Y, Liu X, Zhang X: The Akt/GSK3beta pathway mediates flurbiprofen-induced neuroprotection against focal cerebral ischemia/reperfusion injury in rats. Biochem Biophys Res Comm 409: 808 - 813, 2011

19. Towfighi A, Ovbiagele B, Saver JL: Therapeutic mile-stone: Stroke declines from the second to the third leading organ- and diseasespecific cause of death in the United States. Stroke 41:499-503, 2009

20. Wang H, Wang G, Yu Y, Wang Y: The role of phosphoinositide-3kinase /Akt pathway in propofol-induced postconditioning against focal cerebral ischemia -reperfusion injury in rats. Brain Res 1297: 177-184, 2009

21. Yip TR, Demaerschalk BM: Estimated cost savings of increased use of intravenous tissue plasminogen activator for acute ischemic stroke in Canada. Stroke 38: 1952-1955, 2007

22. Yuji K, Naoki T, Kazutaka S: Cell therapy for stroke: Emphasis on optimizing safety and efficacy profile of endothelial progenitor cells. Curr Pharm Des 18(25): 3731-3734, 2012

23. Zhang ZG, Zhang L, Jiang Q, Zhang R, Davies K, Powers C, Bruggen Nv, Chopp M: VEGF enhances angiogenesis and promotes blood-brain barrier leakage in the ischemic brain. $\mathrm{J}$ Clin Invest 106:829-838, 2000

24. Zhao H, Sapolsky RM, Steinberg GK: Phosphoinositide-3-kinase/ akt survival signal pathways are implicated in neuronal survival after stroke. Mol Neurobiol 34:249-270, 2006 